\title{
Morphometric Study of Patella and Its Role in Sex Determination among Egyptians Using Magnetic Resonance Imaging
}

\author{
Dalia Alsaied Ahmed ${ }^{1}$, Nehal Tharwat ${ }^{2}$, Nermin M. Emam ${ }^{1}$
}

\begin{tabular}{ll} 
& ABSTRACT \\
KEYWORDS & $\begin{array}{l}\text { In the morphometry of the patella, there are several naturally occurring } \\
\text { Knee, } \\
\text { Patella, }\end{array}$ Imaging, \\
\cline { 2 - 3 } Sex, & $\begin{array}{l}\text { variations. Few studies have focused on these anatomical differences among the Egyptian } \\
\text { population. The present work aimed to evaluate age and gender variations in different }\end{array}$ \\
metermination, \\
Morphometric,
\end{tabular}

\section{Introduction}

The determination of gender is one of the main goals of forensic anthropology in the identification process (Perret et al., 2008). Classification of an individual as either male or female is considered gender determination (Afrianty et al., 2013). If the entire skeleton is available for examination, sex estimation will be more accurate, but in forensic situations, human skeletal remains are often incomplete or destroyed due to taphonomic history, animal behaviour, preservation conditions, or recovery proficiency (Krishan et al., 2016).

\footnotetext{
(1) Forensic Medicine and Clinical Toxicology Department, Faculty of Medicine, Mansoura University, Egypt.

(2) Diagnostic Radiology Department, Faculty of Medicine, Mansoura University, Egypt.
}

Since the skull, pelvis, and long bones are often missing or fractured, sex prediction must depend on other parts of the skeleton. However, the accuracy of sex determination from other skeletal remains depends on the degree of sexual dimorphism shown by the skeleton. Observation of morphological features is generally done on unfragmented skeletal remains (Gonzalez et al., 2009; Amores-Ampuero, 2017), while the metric method or statistical quantitative analysis is more objective, repeatable, and can be applied even on fragmented skeletal remains (Krishan et al., 2016).

In previous studies, other skeletal elements have been used for sex assessment such as long bones (Boldsen et al., 2015; Hishmat et al., 2015), sternum (Yonguc et al., 
2015; Zhang et al., 2016b), scapula (Zhang et al., 2016a; El Morsi et al., 2017), clavicle (Atterton et al., 2016; Torimitsu et al., 2018), ribs (Kubicka and Piontek, 2016), fingers (Karn et al., 2019) and talus (Mahakkanukrauh et al., 2014). The patella which is the largest sesamoid bone that develops in the muscletendon of the quadriceps femoris has great significance nowadays not only for surgical orthopedic operations and designing of patellar prosthesis but also in forensic evaluations, evolutionary biology, and morphometric analysis. It resists postmortem and taphonomic changes, therefore it is useful for sex determination when complete skeletal remains are not present (Vohra., 2017).

While most skeleton features have been subjected to discriminant function analysis for sex estimation, the patella has received little attention. More studies from different populations are needed to encourage the usage of patellar parameters "at least one patella" in forensic anthropology for sex determination from incomplete skeletons retaining (Peckmann and Fisher, 2018; Michiue et al., 2018).

By using non-ionizing radiation, magnetic resonance imaging (MRI) allows for fast, complete, and clear visualization of the entire patellofemoral joint. Diagnostic arthroscopy has been replaced as a primary diagnostic modality by MRI, which is now considered a standard procedure. Furthermore, anatomical variations that may lead to patellar dysfunction and other types of patellofemoral joint disorders can be assessed using MRI (Muhamed and Saralaya, 2017).

The present work aims to evaluate the gender and age differences in the patella's different morphometric parameters to the greatest possible accuracy using MRI to determine the percentage of accurate sex prediction based solely on patellar measurements among the Egyptian population aged $20-70$ years.

\section{Materials and Methods:}

The materials needed for the current study include MR images of the subjects' knee joints (right or left), obtained retrospectively from the picture archiving and communication system (PACS), confirming the inclusion and exclusion requirements, collected from the Radiology Department, Faculty of Medicine, Mansoura University. The research protocol was approved by Institutional Research Board (IRB), Faculty of Medicine, Mansoura University (R. 21.01.1145.R1.R2).

Study Design: The current study is a retrospective study that was performed from January 2019 to December 2020 on 200 MR images of normal patellae (100 males and 100 females).

- Inclusion Criteria: The study included randomly selected knee joint MR photographs taken for different purposes in the Radiology Department from subjects with known sex and their age ranging from 20 - 70 years.

- Exclusion Criteria: Subjects with a history of congenital abnormalities in the knee, edema, surgery, severe trauma, knee joint tumor, acute patellar dislocation, rheumatoid arthritis, and any pathological changes were excluded from the study. Extensive bone erosions and loss of bone density were also excluded.

\section{Materials Used (MRI Protocol):}

The patellar parameters in the study were measured from MR images taken from the PACS of the Radiology Department with the parameters mentioned below: (1) All studies were done using a 1.5 Tesla scanner (Philips Ingenia). (2) Every patient lies in a supine position with knee joint straightened and neutrally rotated at the time of imaging. (3) T2-weighted turbo spin-echo axial and sagittal 
images of the knee joint with the following parameters (repetition time (TR) $\geq 2000$, echo time (TE) 80-100, a field of view (FOV) 1617 , matrix $256 \times 256$, slice thickness $3 \mathrm{~mm}$ ) were studied.

To determine the anatomical morphology of the patella., the following nine parameters were measured by an expert radiologist (15 years experience in musculoskeletal imaging) on the patellar mid axial and sagittal MR images, where the patellar maximal diameters were visible (Muhamed and Saralaya, 2017). The cartilage was not included in the measurements when the chondral surface was involved in any parameter measurement, due to age-based wear and tear variations in the measurements of the cartilage.

The parameters include: (1) Patella Angle "PA": It is the angle between medial and lateral patellar facets with the point of patellar central ridge as the zenith, (2) Patella Width "PW": It is the maximum distance between the most medial and the most lateral patellar points, (3) Patella Height "PH": It is the maximum distance between patellar apex tip and the base, (4) Patella Thickness "PT": It is the distance between the points of patellar central ridge to patellar anterior point, (5) Patella Lateral Facet Width "PLFW": It is the distance between the most lateral point of the patella and the patellar central point, (6) Patella Facet Thickness "PFT": It is the distance between patellar central point and patellar central ridge point, (7) Patella Lateral Facet Ratio "PLFR=PLFW/PW": It is patella lateral facet width : patella width, (8) Patella Relative Thickness "PRT=PT/PW": It is patella thickness : patella width and (9) Patella Facet Thickness Ratio "PFTR=PFT/PT": It is patella facet thickness : patella thickness.

All measurements were obtained using the secondary workstation provided by the vendor. To assess sex and age variations concerning patellar measurements; a total of 200 patellar MR images were divided into four groups based on their age., 20-30, 31-40, 4150 , and $>50$ years.

\section{Statistical Analysis:}

Data were fed to the computer and analyzed using "IBM SPSS Corp." released in 2013. IBM SPSS Statistics for Windows, Version 22.0. Armonk, NY: IBM Corp. Qualitative data were described using number and percent. Quantitative data were described using the mean, the standard deviation for parametric data after testing normality using the "Kolmogrov-Smirnov test". The significance of the obtained results was judged at the (0.05) level. "Student t-test" was used to compare 2 independent groups of parametric variables. Discriminant Analysis was used to predict membership in two or more mutually exclusive groups. "Pearson correlation" was used to correlate continuous parametric variables. One Way "ANOVA test" was used to compare more than 2 independent groups of parametric continuous variables with the "Post Hoc Tukey test" for within-group significance (Borror, 2009).

\section{Results:}

Two hundred knee MR images of patients; $100(50 \%)$ were males and $100(50 \%)$ were females; during the period (2019-2020) were included in this retrospective study. They were divided into four different age groups, "20-30", "31-40", "41-50", and ">50" years. The total number of subjects are $53(26.5 \%)$, 45 (22.5\%), 51 (25.5\%), 51 (25.5\%) subjects respectively in each group of age, as illustrated in table (1). 
Table (1): Number and percentage of subjects in each group of age:

\begin{tabular}{cc}
\hline Age / years & Total number of subjects (\%) \\
\hline $20-30$ & $53(26.5) \%$ \\
$31-40$ & $45(22.5) \%$ \\
$41-50$ & $51(25.5) \%$ \\
$>50$ & $51(25.5) \%$ \\
\hline Total & $200(100) \%$ \\
\hline
\end{tabular}

The measurement values related to morphological parameters of the patella are shown in table (2). The results of the current study showed that the average size of the
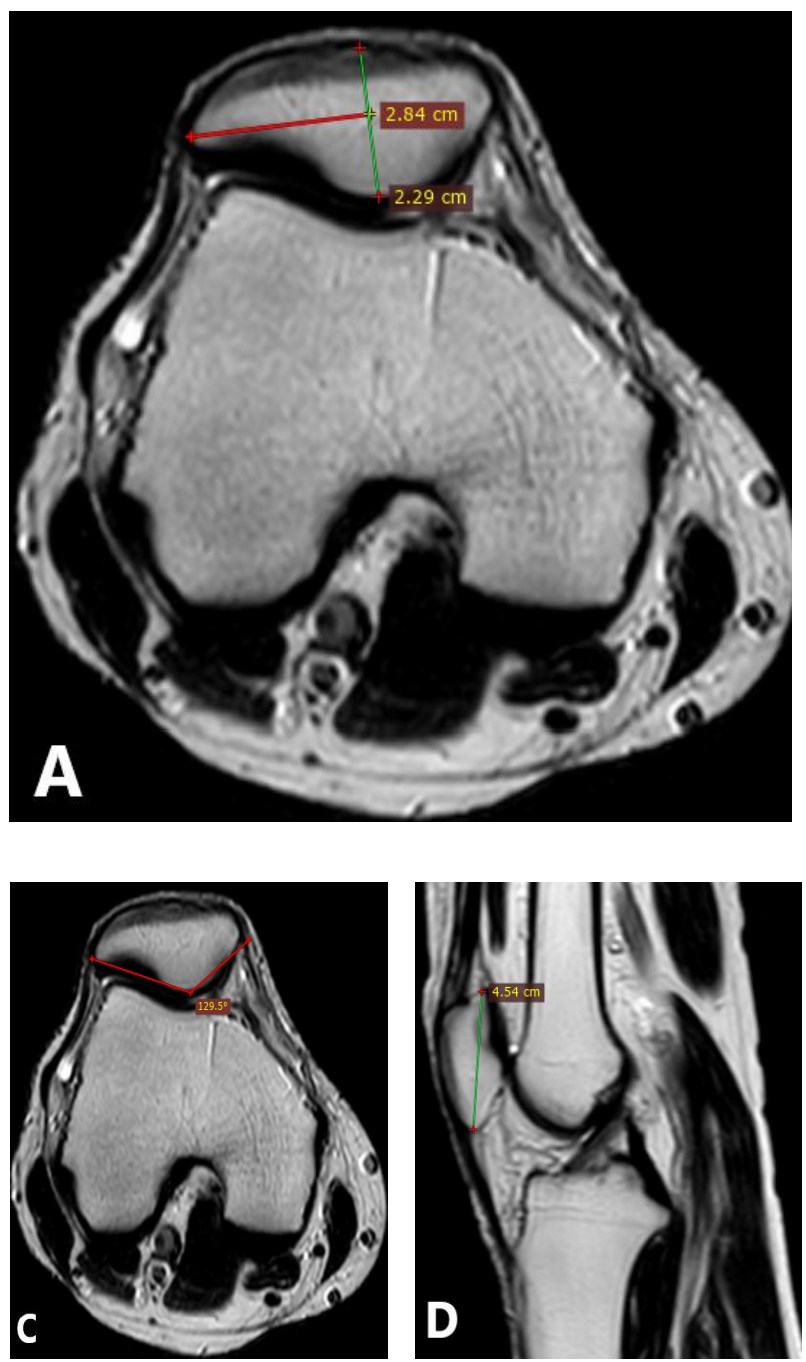

male patella is larger than that of the female, which was statistically significant (Figures 1, 2).

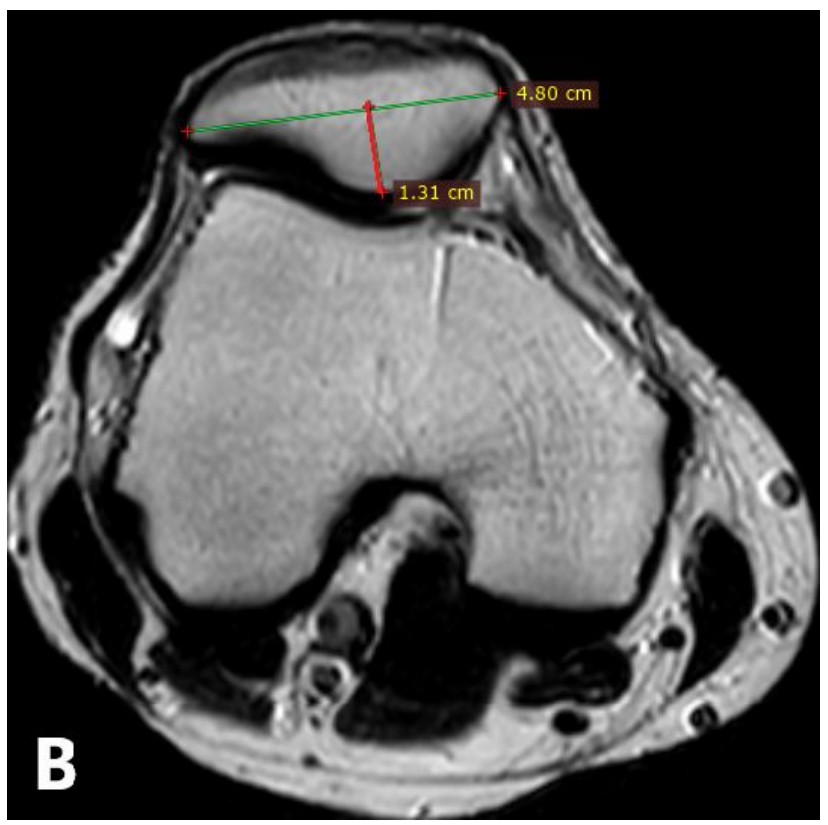

Fig. (1): Male patient 35 years old right knee MRI (A, B and $\mathrm{C}$ axial, $\mathrm{D}$ sagittal $\mathrm{T} 2$ images) showing: A) Patellar thickness (green line) $2.29 \mathrm{~cm}$, patellar lateral facet width (red line) $2.84 \mathrm{~cm}$. B) patellar width (green line) 4.8 $\mathrm{cm}$, patellar facet thickness (red line) 1.31 $\mathrm{cm}$. C) Patellar angle 129.5 degrees. D) Patellar height $4.54 \mathrm{~cm}$. 

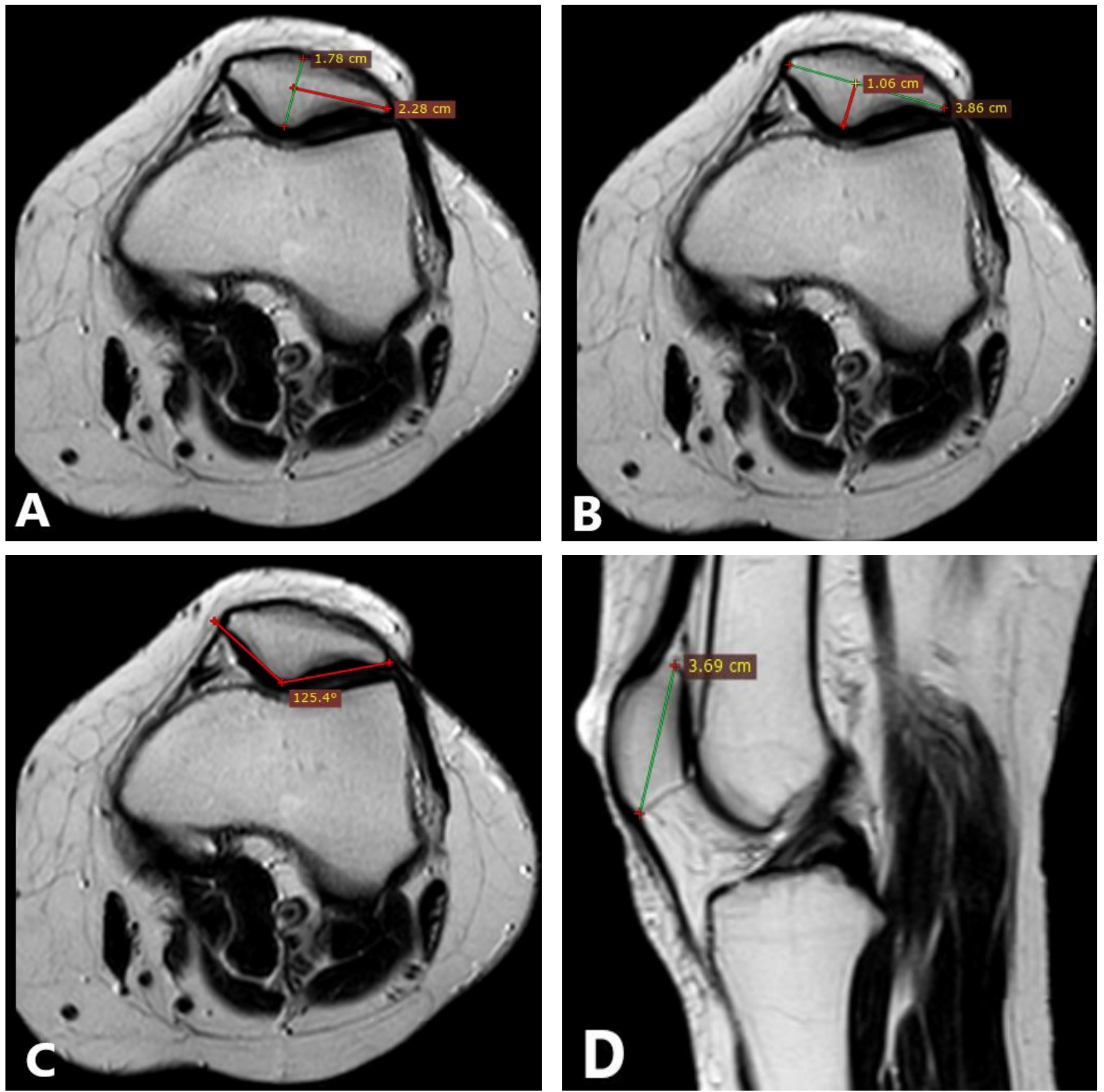

Fig. (2): Female patient 51 years old left knee MRI (A, B and C axial, D sagittal T2 images) showing: A) Patellar thickness (green line) $1.78 \mathrm{~cm}$, patellar lateral facet width (red line) $2.28 \mathrm{~cm}$. B) patellar width (green line) $3.86 \mathrm{~cm}$, patellar facet thickness (red line) 1.06 $\mathrm{cm}$. C) Patellar angle 125.4 degrees. D) patellar height $3.69 \mathrm{~cm}$.

There was a gender-specific difference in patellar measurements. There was a statistically significant difference $(\mathrm{p} \leq 0.05)$ in the mean values of patellar parameters among gender in all age groups for 5 out of the 9 parameters indicating sex differences in the patellar measurements through the Egyptian population as shown in table (2). 
Table (2): Mean patellar measurements distribution according to age and sex of the studied cases:

\begin{tabular}{|c|c|c|c|c|c|}
\hline Age / years & Patellar parameters & Male & Female & $t$ - test & p value \\
\hline \multirow{9}{*}{ 20-30 } & $\mathrm{PH}$ & $4.54 \pm 0.322$ & $3.81 \pm 0.22$ & 8.02 & $<0.001 *$ \\
\hline & PA & $134.51 \pm 4.99$ & $130.06 \pm 4.17$ & 3.05 & $0.004 *$ \\
\hline & PW & $4.57 \pm 0.31$ & $3.94 \pm 0.29$ & 6.80 & $<0.001^{*}$ \\
\hline & PT & $2.06 \pm 0.22$ & $1.68 \pm 0.26$ & 5.38 & $<0.001^{*}$ \\
\hline & PLFW & $2.59 \pm 0.19$ & $2.16 \pm 0.28$ & 6.29 & $<0.0001^{*}$ \\
\hline & PFT & $1.10 \pm 0.12$ & $0.919 \pm 0.14$ & 4.62 & $<0.001^{*}$ \\
\hline & PLFR & $0.567 \pm 0.031$ & $0.545 \pm 0.046$ & 1.98 & 0.054 \\
\hline & PRT & $0.449 \pm 0.039$ & $0.422 \pm 0.054$ & 2.05 & $0.045^{*}$ \\
\hline & PFTR & $0.535 \pm 0.052$ & $0.549 \pm 0.059$ & 0.847 & 0.401 \\
\hline \multirow{9}{*}{$31-40$} & $\mathrm{PH}$ & $4.52 \pm 0.45$ & $3.85 \pm 0.22$ & 6.98 & $<0.001 *$ \\
\hline & $\mathrm{PA}$ & $133 \pm 4.20$ & $126.95 \pm 4.95$ & 4.54 & $<0.001 *$ \\
\hline & PW & $4.59 \pm 0.31$ & $3.96 \pm 0.25$ & 8.07 & $<0.001^{*}$ \\
\hline & PT & $2.12 \pm 0.20$ & $1.79 \pm 0.25$ & 4.98 & $<0.001 *$ \\
\hline & PLFW & $2.48 \pm 0.29$ & $2.20 \pm 0.17$ & 4.22 & $<0.001^{*}$ \\
\hline & PFT & $1.11 \pm 0.15$ & $0.98 \pm 0.13$ & 3.21 & $0.002 *$ \\
\hline & PLFR & $0.539 \pm 0.052$ & $0.537 \pm 0.106$ & 0.120 & 0.905 \\
\hline & PRT & $0.461 \pm 0.04$ & $0.451 \pm 0.051$ & 0.713 & 0.479 \\
\hline & PFTR & $0.520 \pm 0.056$ & $0.549 \pm 0.076$ & 1.48 & 0.145 \\
\hline \multirow{9}{*}{$41-50$} & $\mathrm{PH}$ & $4.56 \pm 0.28$ & $3.83 \pm 0.26$ & 9.28 & $<0.001^{*}$ \\
\hline & $\mathrm{PA}$ & $133 \pm 3.45$ & $132.25 \pm 5.12$ & 0.59 & 0.555 \\
\hline & PW & $4.56 \pm 0.32$ & $4.07 \pm 0.29$ & 5.46 & $<0.001^{*}$ \\
\hline & PT & $2.07 \pm 0.17$ & $1.77 \pm 0.19$ & 5.69 & $<0.001^{*}$ \\
\hline & PLFW & $2.62 \pm 0.19$ & $2.23 \pm 0.23$ & 6.26 & $<0.001^{*}$ \\
\hline & PFT & $1.148 \pm 0.15$ & $0.97 \pm 0.15$ & 4.04 & $<0.001^{*}$ \\
\hline & PLFR & $0.575 \pm 0.046$ & $0.547 \pm 0.041$ & 2.18 & $0.035^{*}$ \\
\hline & PRT & $0.455 \pm .032$ & $0.433 \pm 0.042$ & 1.97 & 0.055 \\
\hline & PFTR & $0.554 \pm 0.068$ & $0.546 \pm 0.056$ & 0.411 & 0.683 \\
\hline \multirow{9}{*}{$>\mathbf{5 0}$} & $\mathrm{PH}$ & $4.36 \pm 0.26$ & $3.89 \pm 0.25$ & 6.51 & $<0.001 *$ \\
\hline & PA & $132.84 \pm 4.93$ & $128.95 \pm 5.47$ & 2.54 & $0.014^{*}$ \\
\hline & PW & $4.52 \pm 0.24$ & $3.96 \pm 0.26$ & 7.74 & $<0.001 *$ \\
\hline & PT & $2.02 \pm 0.18$ & $1.85 \pm 0.18$ & 3.17 & $0.0003^{*}$ \\
\hline & PLFW & $2.56 \pm 0.27$ & $2.15 \pm 0.19$ & 6.31 & $<0.001 *$ \\
\hline & PFT & $1.12 \pm 0.12$ & $1.01 \pm 0.129$ & 2.93 & $0.005^{*}$ \\
\hline & PLFR & $0.566 \pm 0.048$ & $0.540 \pm 0.038$ & 2.08 & $0.043 *$ \\
\hline & PRT & $0.445 \pm 0.032$ & $0.468 \pm 0.044$ & 1.89 & 0.065 \\
\hline & PFTR & $0.555 \pm 0.044$ & $0.547 \pm 0.055$ & 0.502 & 0.618 \\
\hline
\end{tabular}

Parameters described as mean \pm SD, the test used: Student t-test, PH: "Patella Height"; PA: "Patella Angle"; PW: "Patella Width"; PT: "Patella Thickness"; PLFW: "Patella Lateral Facet Width"; PFT: "Patella Facet Thickness"; PLFR: "Patella Lateral Facet Ratio"; PRT: "Patella Relative Thickness"; PFTR: "Patella Facet Thickness Ratio". *statistically significant if p $(\leq 0.05)$.

Through all patellar parameters, $\mathrm{PH}$, PW, PT, PLFW, and PFT showed statistically significant sexual dimorphism $(\mathrm{p} \leq 0.05)$ in all age groups. In all age groups, the only patellar parameter which was not significant statistically was PFTR. Moreover, PA was insignificant in the (41-50) age group, PLFR was insignificant in (20-30) and (31-40) age groups and PRT was insignificant in (31-40), (41-50), and (>50) age groups.
Table (3) shows the discriminate loading of the patellar measurements in each age group. $\mathrm{PH}$ was a highly discriminating parameter in (20-30) and (41-50) age groups followed by PW in (20-30) age group and the PLFW in (41-50) age group. However, in (31$40)$ and $(>50)$ age groups the PW was a highly discriminating parameter followed by the PH. 
Table (3): Summary of interpretive measures for discriminant analysis:

\begin{tabular}{|c|c|c|c|c|c|}
\hline Age/ years & Patellar parameters & Unstandardized & Standardized & $\begin{array}{l}\text { Discriminate } \\
\text { Loading }\end{array}$ & $\begin{array}{l}\text { Univariate } \\
\text { F ratio }\end{array}$ \\
\hline \multirow{12}{*}{ 20-30 } & $\mathrm{PH}$ & .070 & .535 & $.757(1)$ & $64.238 * *$ \\
\hline & PA & 3.542 & .333 & $0.288(6)$ & $9.336 * *$ \\
\hline & PW & -4.586 & 3.805 & $0.642(2)$ & $46.293 * *$ \\
\hline & PT & 1.790 & -3.453 & $0.508(4)$ & $28.953 * *$ \\
\hline & PLFW & 59.041 & -2.292 & $0.594(3)$ & $39.654 * *$ \\
\hline & PFT & -10.269 & .459 & $0.436(5)$ & $21.382 * *$ \\
\hline & PLFR & 64.462 & 2.133 & $0.187(8)$ & 3.906 \\
\hline & PRT & -14.872 & 2.856 & $0.194(7)$ & $4.214^{*}$ \\
\hline & PFTR & 12.555 & -.250 & $-0.08(9)$ & .718 \\
\hline & Group centroid low & 0.914 & & & \\
\hline & Group centroid high & -2.316 & & & \\
\hline & $\begin{array}{l}\text { Wilks Lambada } \\
\text { (Canonical correlation) }\end{array}$ & $0.312^{*}$ & & & \\
\hline \multirow{12}{*}{$31-40$} & $\mathrm{PH}$ & -.064 & -.377 & $-0.708(2)$ & $48.645 * *$ \\
\hline & PA & -13.511 & -.298 & $-0.460(4)$ & $20.563 * *$ \\
\hline & PW & 27.198 & 1.303 & $-0.819(1)$ & $65.071 * *$ \\
\hline & PT & -1.135 & -2.149 & $-0.505(3)$ & $24.762 * *$ \\
\hline & PLFW & -2.089 & .209 & $-0.429(5)$ & $17.828 * *$ \\
\hline & PFT & .905 & -1.871 & $-0.326(6)$ & $10.284 * *$ \\
\hline & PLFR & 65.307 & -.185 & $-0.012(9)$ & .014 \\
\hline & PRT & -9.287 & 3.148 & $-0.072(8)$ & .508 \\
\hline & PFTR & 4.724 & 1.881 & $0.150(7)$ & 2.19 \\
\hline & Group centroid low & -1.72 & & & \\
\hline & Group centroid high & 1.108 & & & \\
\hline & $\begin{array}{l}\text { Wilks Lambada } \\
\text { (Canonical correlation) }\end{array}$ & $0.336^{*}$ & & & \\
\hline \multirow{12}{*}{ 41-50 } & PH & .011 & .862 & $0.872(1)$ & $86.053 * *$ \\
\hline & $\mathrm{PA}$ & 4.257 & .047 & $0.056(8)$ & .354 \\
\hline & PW & -13.143 & -.897 & $0.513(4)$ & $29.782 * *$ \\
\hline & PT & 3.192 & -.665 & $0.534(3)$ & $32.215^{* *}$ \\
\hline & PLFW & -23.200 & 1.713 & $0.589(2)$ & $39.276^{* *}$ \\
\hline & PFT & 8.171 & .631 & $0.380(5)$ & $16.304^{* *}$ \\
\hline & PLFR & 7.622 & -1.018 & $0.205(6)$ & $4.739 *$ \\
\hline & PRT & -3.635 & .286 & $0.185(7)$ & 3.876 \\
\hline & PFTR & -2.916 & -.835 & $0.039(9)$ & .169 \\
\hline & Group centroid low & 1.455 & & & \\
\hline & Group centroid high & -1.654 & & & \\
\hline & $\begin{array}{l}\text { Wilks Lambada } \\
\text { (Canonical correlation) }\end{array}$ & $0.285^{*}$ & & & \\
\hline \multirow{12}{*}{$>\mathbf{5 0}$} & PH & .013 & .503 & $0.713(2)$ & $42.393 * *$ \\
\hline & $\mathrm{PA}$ & -12.550 & .068 & $0.279(6)$ & $6.469 *$ \\
\hline & PW & 22.357 & -.800 & $0.848(1)$ & $59.842 * *$ \\
\hline & PT & 2.009 & 4.714 & $0.347(4)$ & $10.049 * *$ \\
\hline & PLFW & 23.561 & -1.143 & $0.691(3)$ & $39.775 * *$ \\
\hline & PFT & -5.063 & -1.568 & $0.321(5)$ & $8.564 * *$ \\
\hline & PLFR & -79.828 & .996 & $0.228(7)$ & $4.330^{*}$ \\
\hline & PRT & 25.872 & -3.214 & $-0.207(8)$ & 3.568 \\
\hline & PFTR & -3.160 & 1.160 & $0.055(9)$ & .252 \\
\hline & Group centroid low & 1.659 & & & \\
\hline & Group centroid high & -0.985 & & & \\
\hline & $\begin{array}{l}\text { Wilks Lambada } \\
\text { (Canonical correlation) }\end{array}$ & $0.370^{*}$ & & & \\
\hline
\end{tabular}

PH: "Patella Height"; PA: "Patella Angle"; PW: "Patella Width"; PT: "Patella Thickness"; PLFW: "Patella Lateral Facet Width"; PFT: "Patella Facet Thickness"; PLFR: "Patella Lateral Facet Ratio"; PRT: "Patella Relative Thickness"; PFTR: "Patella Facet Thickness Ratio". *Statistically significant if $\mathrm{p}(\leq 0.05)$. 
On evaluating the mean values of various morphometric patellar measurements along with the four age groups, a statistically significant difference $(\mathrm{p} \leq 0.05)$ was detected in some patellar parameters as shown in table (4). Among all patellar parameters, there was a statistically significant difference $(p \leq 0.05)$ in the following measurements; PH, PA, PW, and PLFW among the four age groups. PA showed the highest statistically significant difference $(p \leq 0.001)$ for age differentiation in all age groups, but no statistically significant difference for PT, PFT, PLFR, PRT, and PFTR among the four age groups.

Table (4): Comparison of patellar measurements among different age groups:

\begin{tabular}{lccccc}
\multicolumn{1}{c}{$\begin{array}{c}\text { Patellar } \\
\text { parameters }\end{array}$} & $20-30$ & \multicolumn{2}{c}{ Age group / years (Mean \pm SD) } \\
PH & $4.33 \pm 0.44^{\mathrm{AB}}$ & $41-40$ & $41-50$ & $>50$ & p value \\
\hline PA & $133.25 \pm 5.48^{\mathrm{AB}}$ & $129.33 \pm 5.48^{\mathrm{AC}}$ & $4.22 \pm 0.46$ & $4.07 \pm 0.34^{\mathrm{B}}$ & $0.012^{*}$ \\
\hline PW & $4.39 \pm 0.41^{\mathrm{AB}}$ & $4.21 \pm 0.42^{\mathrm{A}}$ & $4.33 \pm 0.39^{\mathrm{C}}$ & $130.39 \pm 5.56^{\mathrm{BD}}$ & $<0.001^{*}$ \\
\hline PT & $1.95 \pm 0.29$ & $1.92 \pm 0.28$ & $1.93 \pm 0.37^{\mathrm{BC}}$ & $0.016^{*}$ \\
PLFW & $2.47 \pm 0.29^{\mathrm{AB}}$ & $2.31 \pm 0.27^{\mathrm{AD}}$ & $2.44 \pm 0.28^{\mathrm{CD}}$ & $1.91 \pm 0.19$ & 0.89 \\
\hline PFT & $1.05 \pm 0.15$ & $1.03 \pm 0.15$ & $1.07 \pm 0.17$ & $1.052 \pm 0.13$ & $0.004^{*}$ \\
\hline PLFR & $0.561 \pm 0.037$ & $0.538 \pm 0.087$ & $0.562 \pm 0.04$ & $0.549 \pm 0.04$ & 0.714 \\
\hline PRT & $0.442 \pm 0.045$ & $0.455 \pm 0.047$ & $0.444 \pm 0.038$ & $0.459 \pm 0.04$ & 0.114 \\
\hline PFTR & $0.539 \pm 0.054$ & $0.538 \pm 0.069$ & $0.550 \pm 0.062$ & $0.550 \pm 0.05$ & 0.140 \\
\hline
\end{tabular}

Used test: One Way ANOVA test, similar superscripted letters denote significant difference between groups having the same letters in the same row by Post Hoc Tukey test. PH: "Patella Height"; PA: "Patella Angle"; PW: "Patella Width"; PT: "Patella Thickness"; PLFW: "Patella Lateral Facet Width"; PFT: "Patella Facet Thickness"; PLFR: "Patella Lateral Facet Ratio"; PRT: "Patella Relative Thickness"; PFTR: "Patella Facet Thickness Ratio". *Statistically significant if p $(\leq 0.05)$.

Moreover, PH, PW, and PLFW were found to be decreasing with age with statistically significant negative correlation $(\mathrm{r}$ $=-0.216,-0.154$, and -0.164 , respectively) when the patellar measurements were analyzed using "Karl Pearson correlation". The remaining patellar parameters had no statistically significant correlation with age as shown in table (5).

Table (5): Correlation between age and patellar measurements among studied cases:

\begin{tabular}{|c|c|c|}
\hline Patellar parameters & Pearson correlation coefficient & Age/years \\
\hline \multirow{2}{*}{ 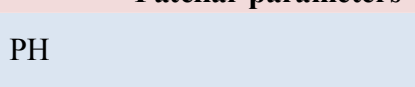 } & $\mathrm{R}$ & $-.216 * *$ \\
\hline & $\mathrm{P}$ & .002 \\
\hline \multirow{2}{*}{ PA } & $\mathrm{R}$ & -.090 \\
\hline & $\mathrm{P}$ & .204 \\
\hline \multirow{2}{*}{ PW } & $\mathrm{R}$ & $-.154 *$ \\
\hline & $\mathrm{P}$ & .029 \\
\hline \multirow{2}{*}{ PT } & $\mathrm{R}$ & -.062 \\
\hline & $\mathrm{P}$ & .379 \\
\hline \multirow{2}{*}{ PLFW } & $\mathrm{R}$ & $-.164 *$ \\
\hline & $\mathrm{P}$ & .020 \\
\hline \multirow{2}{*}{ PFT } & $\mathrm{R}$ & .014 \\
\hline & $\mathrm{P}$ & .843 \\
\hline \multirow{2}{*}{ PLFR } & $\mathrm{R}$ & -.030 \\
\hline & $\mathrm{P}$ & .668 \\
\hline \multirow{2}{*}{ PRT } & $\mathrm{R}$ & .092 \\
\hline & $\mathrm{P}$ & .191 \\
\hline \multirow{2}{*}{ PFTR } & $\mathrm{R}$ & .082 \\
\hline & $\mathrm{P}$ & .245 \\
\hline
\end{tabular}

r: Pearson correlation coefficient, PH: "Patella Height"; PA: "Patella Angle"; PW: "Patella Width"; PT: "Patella Thickness"; PLFW: "Patella Lateral Facet Width"; PFT: "Patella Facet Thickness"; PLFR: "Patella Lateral Facet Ratio"; PRT: "Patella Relative Thickness"; PFTR: "Patella Facet Thickness Ratio". *Significant p-value " $\leq 0.05$ ”. **Highly significant p-value " $\leq 0.001$ ”. 
Table (6) shows that the highest average accuracy of patellar measurements in differentiating sex was in the (41-50) age group with $93.6 \%$ accuracy followed by $(20-$ 30) age group with $90.6 \%$ accuracy.

Table (6): Average accuracy of patellar measurements in differentiating sex at different age groups:

\begin{tabular}{|c|c|c|c|c|c|}
\hline \multirow{2}{*}{$\begin{array}{c}\text { Age/ } \\
\text { years }\end{array}$} & \multirow{2}{*}{$\begin{array}{c}\text { Patellar } \\
\text { parameters }\end{array}$} & \multicolumn{2}{|c|}{ Classification function coefficient (Sex) } & \multirow{2}{*}{$\begin{array}{c}\text { Corrected } \\
\text { classification } \\
(\%)\end{array}$} & \multirow{2}{*}{$\begin{array}{c}\text { Average accuracy } \\
(\%)\end{array}$} \\
\hline & & Male & Female & & \\
\hline \multirow{10}{*}{$20-30$} & PA & 18.768 & 18.543 & \multirow{10}{*}{$92.5 \%$} & \multirow{10}{*}{$90.6 \%$} \\
\hline & PFT & -6772.858 & -6784.301 & & \\
\hline & PFTR & 12860.121 & 12874.937 & & \\
\hline & $\mathrm{PH}$ & -171.349 & -177.130 & & \\
\hline & PLFR & 24632.336 & 24441.609 & & \\
\hline & PLFW & -5353.847 & -5320.673 & & \\
\hline & PRT & 15935.088 & 15726.852 & & \\
\hline & PT & 289.797 & 337.838 & & \\
\hline & PW & 4655.639 & 4615.080 & & \\
\hline & (Constant) & -15148.897 & -14891.637 & & \\
\hline \multirow{10}{*}{$31-40$} & PA & 2.208 & 2.026 & \multirow{10}{*}{$94.1 \%$} & \multirow{10}{*}{$88.2 \%$} \\
\hline & PFT & -4869.765 & -4907.940 & & \\
\hline & PFTR & 9081.527 & 9158.371 & & \\
\hline & $\mathrm{PH}$ & 24.455 & 21.247 & & \\
\hline & PLFR & 216.679 & 210.779 & & \\
\hline & PLFW & -185.576 & -183.018 & & \\
\hline & PRT & 18615.725 & 18800.242 & & \\
\hline & PT & -1982.994 & -2009.233 & & \\
\hline & PW & 2080.257 & 2093.604 & & \\
\hline & (Constant) & -6655.560 & -6711.923 & & \\
\hline \multirow{10}{*}{$41-50$} & $\mathrm{PA}$ & 7.534 & 7.500 & \multirow{10}{*}{$97.9 \%$} & \multirow{10}{*}{$93.6 \%$} \\
\hline & PFT & -847.348 & -860.586 & & \\
\hline & PFTR & 605.424 & 646.291 & & \\
\hline & $\mathrm{PH}$ & 102.471 & 92.545 & & \\
\hline & PLFR & 22583.750 & 22655.889 & & \\
\hline & PLFW & -5315.860 & -5341.266 & & \\
\hline & PRT & 31804.369 & 31780.668 & & \\
\hline & PT & -7162.360 & -7151.057 & & \\
\hline & $\mathrm{PW}$ & 6060.868 & 6069.936 & & \\
\hline & (Constant) & -13577.058 & -13568.610 & & \\
\hline \multirow{10}{*}{$>50$} & $\mathrm{PA}$ & -8.864 & -8.898 & \multirow{10}{*}{$90.2 \%$} & \multirow{10}{*}{$84.3 \%$} \\
\hline & PFT & -9052.919 & -9019.742 & & \\
\hline & PFTR & 16443.648 & 16384.545 & & \\
\hline & $\mathrm{PH}$ & -88.994 & -94.305 & & \\
\hline & PLFR & 13245.004 & 13182.719 & & \\
\hline & PLFW & -3200.557 & -3187.173 & & \\
\hline & PRT & 18066.735 & 18277.762 & & \\
\hline & PT & 732.887 & 664.494 & & \\
\hline & PW & 3879.062 & 3887.416 & & \\
\hline & (Constant) & -11904.490 & -11876.974 & & \\
\hline
\end{tabular}

PH: "Patella Height"; PA: "Patella Angle"; PW: "Patella Width"; PT: "Patella Thickness"; PLFW: "Patella Lateral Facet Width"; PFT: "Patella Facet Thickness"; PLFR: "Patella Lateral Facet Ratio"; PRT: "Patella Relative Thickness"; PFTR: "Patella Facet Thickness Ratio". 


\section{Discussion:}

In forensic anthropology, gender determination is considered an important issue, because it is the first step in the identification process. The patella is made up of highly complex trabecular bone. There is a scarcity of data on gender and age-related anatomical comparisons of patellar measurements (Afrianty et al., 2013).

Direct and indirect measurements can be used to determine patella morphometry. The direct calculation is done with dried bone, skeletal remains, or intraoperative measurements taken with a caliper by the surgeon, which takes time and is subject to observer variability which might reduce the prediction accuracy (Akhlaghi et al., 2010; Muhamed et al., 2017). Indirect measurement is a method of measuring radiographic images that is simple, inexpensive, and noninvasive, as well as providing digital data for future study and reference. Several studies have adapted the use of X-ray, Computed Tomography, and MRI in the evaluation of patellar morphometry (Peng et al., 2014; Teke et al., 2018; Jain et al., 2019; Zhan et al., 2020).

The MR films are a non-invasive, fast, reliable, and inexpensive method to use (Ottow et al., 2017). MRI comes into consideration as a radiation-free imaging method to determine skeletal age, gender, and other forensic medicine applications, such as postmortem imaging (Daghighi et 1., 2021). Furthermore, recent research has adapted the use of a fully automated and computer-driven approach for estimating age based on threedimensional (3D) knee MRIs using machine learning (artificial intelligence) (Dallora et al., 2019; Mauer et al., 2020). Our study has observed several significant patellar anatomic differences concerning gender and age among the Egyptian population using MRI.
Regarding gender variation, most of the patellar parameters measured on MRI of the Egyptian population exhibited statistically significant variations between both males and females. The male patellae were found to have a greater geometrical dimension than the female patellae. In the present study, most of the patellar measurements' mean values were higher in males than females. These results suggest that the patella in this population is sexually dimorphic.

Similarly, it was found by Peckmann et al. (2016) in the Spanish population, Vohra (2017), Muhamed et al. (2017) and Jain et al. (2019) in the Indian population, Peng et al. (2014), Shang et al. (2014) and Zhan et al. (2020) in Chinese population, Peckmann and Fisher (2018) in African American, Teke et al. (2018) in Turkish population, Akhlaghi et al. (2010) and Rahmani et al. (2020) in Iranian population.

In this study, the patellar measurements were obtained from the MRI of 200 patients of the Egyptian Population, 5 out of the 9 measurements in all age groups were statistically significant, indicating sex differences in the patellar measurements $(\mathrm{p} \leq$ 0.05 ). These five patellar measurements were PH, PW, PT, PLFW, and PFT. On the other hand, the patellar measurement which was not significant statistically in all age groups was the PFTR. Moreover, PA was insignificant in the (41-50) age group, PLFR was insignificant in (20-30) and (31-40) age groups and PRT was insignificant in (31-40), (41-50), and ( $>50)$ age groups.

This is in agreement with Muhamed et al. (2017) who reported that PW, PT, PLFW, and PFT showed highly statistically significant sexual dimorphism $(\mathrm{p} \leq 0.001)$. However, PLFR was not statically significant.

The results of the current study demonstrated high accuracy rates for sex 
determination in the Egyptian population from the patella (84.3\%-93.6) in different age groups. The highest average accuracy of patellar measurements in differentiating sex was in the (41-50) age group with 93.6\% accuracy followed by the (20-30) age group with $90.6 \%$ accuracy.

Similarly, Zhan et al. (2020) reported $81.9 \%-91.6 \%$ sex estimation accuracy rates among the Chinese population using a combination of the patellar parameters. Also, Teke et al. (2018) reported 87-91\% accuracy when estimating sex using morphological analysis of the patella in the Turkish population. Peckmann and Fisher (2018) study among African Americans and Rahmani et al. (2020) study among the Iranian population reported lower sex estimation accuracy rates when using patella metric indicators (80-85\% and $83 \%$ respectively).

Regarding age variations, this study demonstrated differences in the anatomical profile of patellar measurements along with age groups. $\mathrm{PH}$ was a highly discriminating parameter in (20-30) and (41-50) age groups followed by PW in (20-30) age group and PLFW in (41-50) age group. However, in (31$40)$ and (>50) age groups, PW was a highly discriminating parameter followed by $\mathrm{PH}$.

Through all the patellar measurements, significant age variations were observed in 4 out of the 9 parameters $(\mathrm{PH}, \mathrm{PA}, \mathrm{PW}$, and PLFW). PA showed the highest statistically significant difference $(p \leq 0.001)$ for age differentiation in all age groups, but no statistically significant difference for PT, PFT, PLFR, PRT, and PFTR. Moreover, PH, PW, and PLFW showed a negative correlation with the age but the remaining patellar parameters did not differ significantly with age. Also, Muhamed and Saralaya (2017) reported that a significant age variation was discovered in PW, PA, PFT, and PLFR, but not for PT, PLFW, PRT, and PFT.

\section{Conclusion:}

Patellar measurements obtained noninvasively from knee MR images revealed gender-specific differences with high accuracy for sex determination useful for forensic purposes. The results of the current MRI analysis on 200 Egyptian males and females revealed that 5 of the 9 morphometric patellar measurements showed substantial gender variance. Significant age variation was found in 4 out of the 9 parameters. This study can provide guidelines for sex and age prediction among Egyptians.

\section{Recommendations:}

Further studies in this field are recommended on a larger population in different governorates of Egypt.

\section{Acknowledgement:}

The authors wish to express their full gratitude to the technicians of the Radiology Department of Mansoura University Hospitals.

\section{Conflict Of Interest :}

All authors confirm that there is no organization support for the present work and there are not any financial connections with any organizations that may have an interest in the current work submitted. 


\section{References:}

Afrianty, I., Nasien, D., Kadir, M., et al. (2013), 'Determination of gender from pelvic bones and patella in forensic anthropology: A comparison of classification techniques. Proceedings 1st International Conference on Artificial Intelligence, Modelling and Simulation'. Artificial Intelligence, Modelling \& Simulation. p. 3-7. https//doi: 10.1109/AIMS.2013.9.

Akhlaghi, M., Sheikhazadi, A., Naghsh, A., et al. (2010), 'Identification of sex in Iranian population using patella dimensions'. Journal of Forensic and Legal Medicine. 17, p.150-155. https://doi:10.1016/j.jflm.2009.11.005.

Amores-Ampuero, A. (2017), 'Sexual dimorphism in base of skull'. Anthropologischer Anzeiger. 74, p.9-14. https//doi:10.1127/anthranz/2017/0603.

Atterton, T., Groote, I.D., and Eliopoulos, C. (2016), 'Assessing size and strength of the clavicle for its usefulness for sex estimation in a British medieval sample'. Journal of Comparative Human Biology. p. 67: 409416.

https://doi:10.1016/j.jchb.2016.09.001.

Boldsen, J.L., Milner, G.R. and Boldsen, S.K. (2015), 'Sex estimation from modern American humeri and femora, accounting for sample variance structure'. The American Journal of Physical Anthropology. 158, p. 745-750. https://doi.org/10.1002/ajpa.22812.

Borror, C. M. (2009), 'Statistical decision making', in Borror, C. M. (editor).The Certified Quality Engineer Handbook (3rd edition). Milwaukee, WI: ASQ Quality Press. United States of America. p. 418-472. ISBN 978-0-873-89745-7.
Daghighi, M.H., Pourisa, M., JavanpourHeravi, H., et al. (2021), 'Application of knee MRI in forensic age estimation: A retrospective cohort'. Radiography. 27, p. 108-114.

https://doi: 10.1016/j.radi.2020.06.019.

Dallora, A.L., Berglund, J.S., Brogren, M., et al. (2019), 'Age Assessment of Youth and Young Adults Using Magnetic Resonance Imaging of the Knee: A Deep Learning Approach'. Journal of Medical Internet Research. 7: e16291. https://doi: 10.2196/16291.

El Morsi, D.A., Gaballah, G., Mahmoud, W., et al. (2017), 'Sex determination in Egyptian population from scapula by computed tomography'. Journal of Forensic Research. 8, p.376.

https://doi:10.4172/2157-7145.1000376.

Gonzalez, P.N., Bernal, V., and Perez, S.I. (2009), Geometric morphometric approach to sex estimation of human pelvis'. Forensic Science International. 189 , p. 68-74.

https//doi:10.1016/j.forsciint.2009.04.012.

Hishmat, A.M., Michiue, T., Sogawa, N., et al. (2015), 'Virtual CT morphometry of lower limb long bones for estimation of the sex and stature using postmortem Japanese adult data in forensic identification'. International Journal of Legal Medicine. 129, p.1173-1182. https://doi: 10.1007/s00414-015-1228-9.

Jain, R., Kalia, R.B., and Das, L. (2019), 'Anthropometric measurements of patella and its clinical implications'. European Journal of Orthopaedic Surgery and Traumatology. 29, p.17651769.

https://doi:10.1007/s00590-019-02490-8. 
Karn, A., Jha, S., Pradhan, A., et al. (2019), 'Determination of gender from the ratio of length of index finger and ring finger (2DL:4DL) in Nepalese adults'. Scholars Journal of Applied Medical Sciences. 7, p. 3480-3484. https://doi:

10.36347/SJAMS.2019.v07i10.053

Krishan, K., Chatterjee, P.M., Kanchan, T., et al. (2016), 'A review of sex estimation techniques during examination of skeletal remains in forensic anthropology casework'. Forensic Science International.261: 165.e1165.e8.https//doi:10.1016/j. forscii nt.2016.02.007.

Kubicka, A.M. and Piontek, J. (2016), 'Sex estimation from measurements of the first rib in a contemporary Polish population'. International Journal of Legal Medicine. 130, p.265-272.

Mahakkanukrauh, P., Praneatpolgrang, S., Ruengdit, S., et al. (2014), 'Sex estimation from the talus in a Thai population'. Forensic Science International. 240, p.e1-e8. https://doi:10.1016/j.forsciint.2014.04.0 01 .

Mauer, M.A., der Well, E.J., Herrmann, J., et al. (2020), 'Automated age estimation of young individuals based on 3D knee MRI using deep learning'. International Journal of Legal Medicine. 35, p. 649-663. https://doi: $10.1007 / \mathrm{s} 00414-020-02465-\mathrm{z}$.

Michiue, T., Hishmat, A.M., Oritani, S, et al. (2018), 'Virtual computed tomography morphometry of the patella for estimation of sex using postmortem Japanese adult data in forensic identification'. Forensic Science International. 285: e1-e6.
Muhamed, R.R. and Saralaya, V.V. (2017), 'Magnetic resonance imaging study of age and sex variation in the anatomy of patellofemoral articulation'.

International Journal of Scientific Research. 9, p.54-65.

https://doi: 10.17354/ijss/2017/555.

Muhamed, R.R, Saralaya, V.V., Murlimanju, B.V., et al. (2017), 'In vivo magnetic resonance imaging morphometry of the patella bone in South Indian population'. Anatomy \& Cell Biology. 50, p. 99-103. https://doi: 10.5115/acb.2017.50.2.99

Ottow, C., Schulz, R., Pfeiffer, H., et al. (2017), 'Forensic age estimation by magnetic resonance imaging of the knee: the definite relevance in bony fusion of the distal femoral- and the proximal tibial epiphyses using closestto-bone T1 TSE sequence'. European Radiology. 27, p. 5041-5048. https://doi: 10.1007/s00330-017-4880-2.

Peckmann, T.R., and Fisher, B. (2018), 'Sex estimation from the patella in an African American population'. Journal of Forensic and Legal Medicine. 54, p. 17.

https://doi: 10.1016/j.jflm.2017.12.002.

Peckmann, T.R., Meek, S., Dilkie, N., et al. (2016), 'Determination of sex from the patella in a contemporary Spanish population'. Journal of Forensic and Legal Medicine. 44, p. 84-91. https://doi:10.1016/j.jflm.2016.09.007.

Peng, S., Linan, Z., Zengtao, H., et al. (2014), 'Morphometric measurement of the patella on 3D model reconstructed from CT scan images for the southern Chinese population'. Chinese Medical Journal.127, p. 96-101. 
Perret, V.A., Staccuni, P., and Quatrehomme, G. (2008), 'Sex determination from the distal part of the femur in a French contemporary population'. Forensic Science International. p. 175:113-117.

https//doi:10.1016/j.forsciint.2007.05.01 8.

\section{Rahmani, E., Mohammadi, S., Babahajian,} et al. (2020), 'Anthropometric characteristics of patella for sex estimation using magnetic resonance images'. Forensic Imaging. 23, p.200412.

https://doi.org/10.1016/j.fri.2020.200412

Shang, P., Zhang, L., Hou, Z., et al. (2014), 'Morphometric measurement of the patella on 3D model reconstructed from CT scan images for the southern Chinese population'. Chinese Medical Journal. 127,. 96-101.

Teke, H.Y., Ünlütürk“,O., Günaydin, E., et al. (2018), 'Determining gender by taking measurements from magnetic resonance images of the patella'. Journal of Forensic and Legal Medicine. 58, p. 87-92.

https://doi:10.1016/j.jflm.2018.05.002.

Torimitsu, S., Makino, Y., Saitoh, H., et al. (2018), 'Sex assessment based on clavicular measurements in a modern Japanese population using multidetector computed tomography'. Forensic Science International. 285: e1-e5. https://doi:10.1016/j.forsciint.2017.10.0 09.
Vohra, P. (2017), "Morphometric study of patella and its role in sex determination". International Journal of Anatomy Physiology and Biochemistry. 4, p. 6-9.

Yonguc, G.N., Kurtulus, A., Bayazit, O., et al. (2015), 'Estimation of stature and sex from sternal lengths: an autopsy study'. Anatomical Science International. 90, p. 89-96.

Zhan, M., Li, C., Fan, F., et al. (2020), 'Estimation of sex based on patella measurements in a contemporary Chinese population using multidetector computed tomography: An automatic measurement method'.Legal Medicine. p.47: 101778 .

https://doi:10.1016/j.legalmed.2020.101 778 .

Zhang, K., Cui, J.H., Luo, Y.Z., et al. (2016a), 'Estimation of stature and sex from scapular measurements by threedimensional volume-rendering technique using in Chinese'. Legal Medicine. 21, p. 58-63. https://doi: 10.1016/j.legalmed.2016.06.004.

Zhang, K., Luo, Y.Z., Chen, X.G., et al. (2016b), 'Sexual dimorphism of sternum using computed tomography - volume rendering technique images of Western Chinese'. Australian Journal of Forensic Sciences. 48, p. 297-304.

https://doi.org/10.1080/00450618.2015. 1060523. 


\title{
دراسة مورفومترية للرضفة ودورها في تحديد الجنس لدى المصريين باستخدام التصوير بالرنين المفناطيسي
}

\author{
داليا السعيد أحمد' ونهال ثروتّ و نيرمين امام' \\ 'قسم الطب الثرعي والسموم الإكلينيكية ـ كليه الطب ـ جامعه المنصورة ـ جمهورية مصر العربية

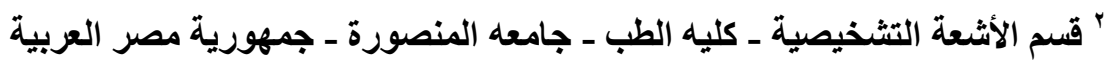

هنالك العديد من التغيرات التي تحدث بشكل طبيعي في شكل الرضفة وقد ركزت القليل من الدراسات على التى

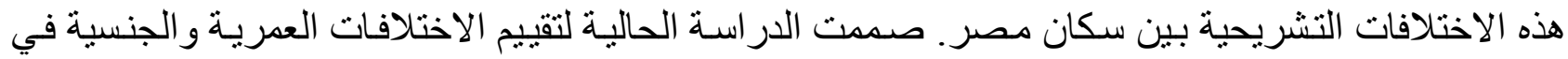

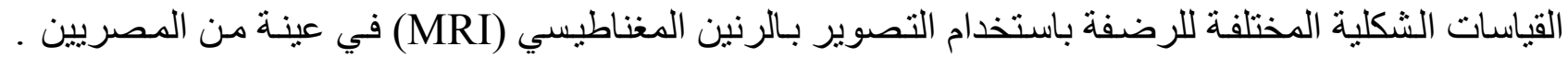

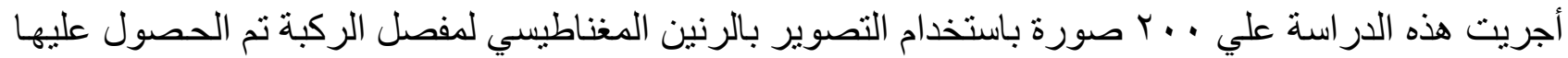

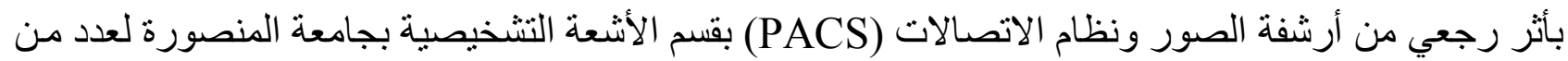

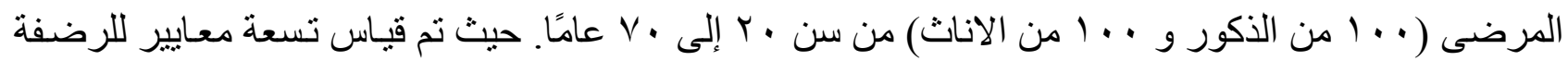

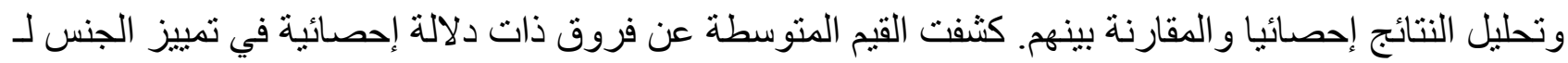

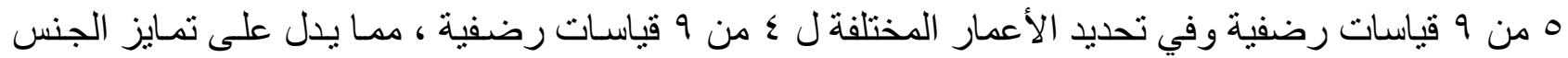

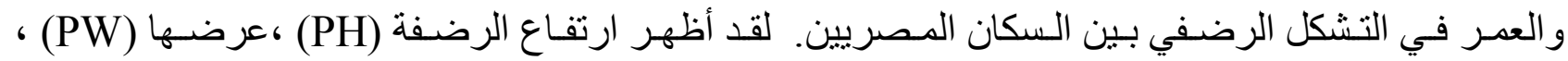

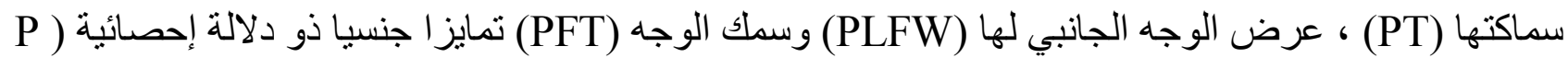

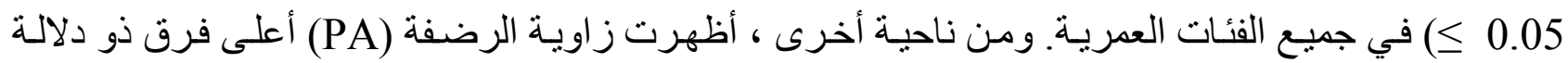

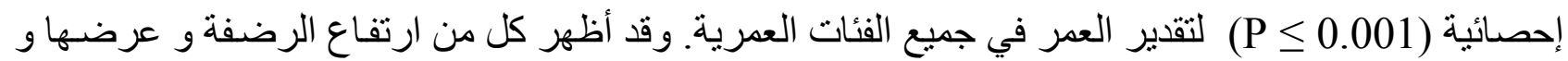

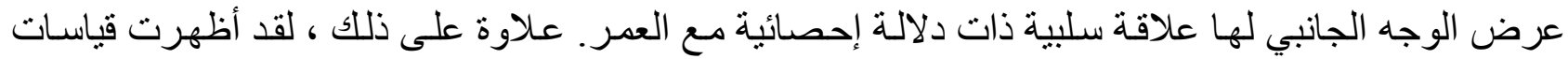

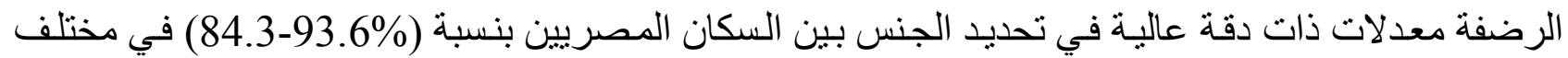

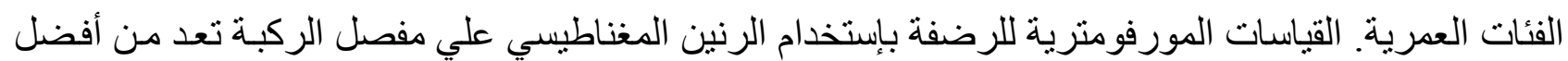
القياسات في تحديد الجنس و التنبؤ بالعمر لدي السكان المصريئ. لرين. 